\title{
FURTHER $q$-SERIES IDENTITIES AND CONJECTURES RELATING FALSE THETA FUNCTIONS AND CHARACTERS
}

\author{
CHRIS JENNINGS-SHAFFER AND ANTUN MILAS
}

\begin{abstract}
In this short note, a companion of [21, we discuss several families of $q$-series identities in connection to false and mock theta functions, characters of modules of vertex algebras, and "sum of tails".
\end{abstract}

\section{INTRODUCTION AND PREVIOUS WORK}

In our previous work [21], motivated by character formulas of vertex algebras and superconformal indices in physics, we obtained various identities for false theta functions including the following elegant identity.

Theorem 1.1. For $k \geq 1$,

$$
\frac{\sum_{n \in \mathbb{Z}} \operatorname{sgn}(n) q^{(k+1) n^{2}+k n}}{(q)_{\infty}^{2 k}}=\sum_{n_{1}, n_{2}, \ldots, n_{2 k-1} \geq 0} \frac{q^{\sum_{i=1}^{2 k-2} n_{i} n_{i+1}+\sum_{i=1}^{2 k-1} n_{i}}}{(q)_{n_{1}}^{2}(q)_{n_{2}}^{2} \cdots(q)_{n_{2 k-1}}^{2}}
$$

where as usual $(a)_{n}=\prod_{i=0}^{n-1}\left(1-a q^{i}\right)$.

We note that these identities have an odd number of summation variables. Interestingly, with an even number of summation variables we obtained a family of modular identities conjectured in [15].

Theorem 1.2. For $k \geq 1$,

$$
\frac{\left(q, q^{2 k+2}, q^{2 k+3} ; q^{2 k+3}\right)_{\infty}}{(q)_{\infty}^{2 k+1}}=\sum_{n_{1}, n_{2}, \ldots, n_{2 k} \geq 0} \frac{q^{\sum_{i=1}^{2 k-1} n_{i} n_{i+1}+\sum_{i=1}^{2 k} n_{i}}}{(q)_{n_{1}}^{2}(q)_{n_{2}}^{2} \cdots(q)_{n_{2 k}}^{2}} .
$$

In a somewhat different direction, in the same paper, we also examined $q$-series identities for false theta functions with half-integral characteristics (here $k \in \mathbb{N}$ and $\epsilon \in\left\{0, \frac{1}{2}\right\}$ )

$$
\frac{\left(-q^{\frac{1}{2}+\epsilon}\right)_{\infty}}{(q)_{\infty}} \sum_{n \in \mathbb{Z}} \operatorname{sgn}(n) q^{\frac{1}{2}(2 k+1)(n+a)^{2}},
$$

for some specific rational numbers $a$. We also considered related identities for certain "shifted" false theta series [21, Section 3].

This paper aims to extend (1.1) and (1.2) in a few directions. Firstly, we would like to study related identities for the false theta functions as in (1.3). Secondly, we relax the condition on the poles in (1.1) and (1.2) and perform a search for identities where the $q$-hypergeometric side takes the form

$$
\sum_{n_{1}, n_{2}, \ldots, n_{k} \geq 0} \frac{q^{n_{1}+n_{2}+\cdots+n_{k}+n_{1} n_{2}+n_{2} n_{3}+\cdots+n_{k-1} n_{k}}}{(q)_{n_{1}}^{r_{1}}(q)_{n_{2}}^{r_{2}} \cdots(q)_{n_{k}}^{r_{k}}},
$$

2020 Mathematics Subject Classification. 11P84,17B69.

A. Milas acknowledges the support from the NSF grant DMS 1601070. 
with $k \leq \sum_{i=1}^{k} r_{i} \leq 2 k$. Lastly, we consider $q$-series identities coming from the formal inversion $q \mapsto q^{-1}$ of the $q$-hypergeometric term in (1.1) and (1.2). This procedure is sometimes used for quantum modular forms to extend a $q$-series defined in the upper half-plane to the lower half-plane.

Our paper is organized as follows. In Sections 2 and 3 we gather several known facts. In Section 4 we prove analogs of Theorems 1.1 and 1.2 for false and classical theta series with half characteristics (Theorem 4.3 and Proposition 4.4). Section 5 is devoted to "inverted identities", under $q \mapsto q^{-1}$, associated to the $q$-hypergeometric series in (1.1) and (1.2). We argue that in both cases we expect modular identities. For the inverted $q$-series coming from (1.2), this is proven in Proposition 5.1 by reduction to the character formula of a principal

subspace of $A_{2 k-1}^{(1)}$. For (1.1), we expect (see Conjecture 5.2) that the resulting inverted series is modular as it is essentially the level one character of the affine vertex algebra $L_{s p(2 k)}\left(\Lambda_{0}\right)$. We show that this is indeed true up to a cubic term (Proposition 5.3). In Section 6, we study more complicated $q$-hypergeometric series of the form (1.4) with $k=2$ and $k=3$. Continuing, in Section 7 we consider identities for the series (1.4) with $r_{i}=1$ for all $i$. For $2 \leq k \leq 8$, except $k=7$, we found several interesting "sums of tails" type identities. Then in Section 8 we connect the $q$-series from Section 7 with characters of modules of principal subspaces and infinite jet schemes. We end with a few remarks for future investigations.

\section{QuAntum Dilogarithm}

2.1. Quantum dilogarithm. As in [21], we will approach several $q$-series identities using the quantum dilogarithm $\phi(x):=\prod_{i \geq 0}\left(1-q^{i} x\right)$. Let $x$ and $y$ be non-commutative variables such that $x y=q y x$, then

$$
\phi(y) \phi(x)=\phi(x) \phi(-y x) \phi(y)
$$

which is Faddeev and Kashaev's pentagon identity for the quantum dilogarithm. This identity implies that

$$
\frac{1}{\phi(x) \phi(y)}=\frac{1}{\phi(y) \phi(-y x) \phi(x)}
$$

where $\frac{1}{\phi\left(x_{1}\right) \phi\left(x_{2}\right) \cdots \phi\left(x_{n}\right)}$ is understood to denote $\frac{1}{\phi\left(x_{1}\right)} \cdot \frac{1}{\phi\left(x_{2}\right)} \cdots \frac{1}{\phi\left(x_{n}\right)}$. For its relevance in $4 \mathrm{~d} / 2 \mathrm{~d}$ dualities in physics see [14, 15] and references therein.

\section{BAILEY'S LEMMA AND OTHER KNOWN q-SERIES IDENTITIES}

As in [21], we require Bailey's lemma and several standard $q$-series identities, which we collect in this section. A pair of sequences $\left(\alpha_{n}, \beta_{n}\right)$ is called a Bailey pair relative to $a$ if

$$
\beta_{n}=\sum_{j=0}^{n} \frac{\alpha_{j}}{(q)_{n-j}(a q)_{n+j}} .
$$

The $k$-fold iteration of Bailey's lemma can be found in its entirety as Theorem 3.4 of [2]. This theorem with $k \mapsto k-1, a=q, b_{1}=b_{2}=\ldots=b_{k-1} \rightarrow \infty, c_{1}=c_{2}=\cdots=c_{k-2}=q$, 
$c_{k-1}=-w^{-1} q, N \rightarrow \infty$, and $n_{j} \mapsto m_{k-j}$ states that

$$
\begin{aligned}
& \sum_{m_{1}, m_{2}, \ldots, m_{k-1} \geq 0} \frac{\left(-w^{-1} q\right)_{m_{1}}(q)_{m_{k-1}}(-1)^{m_{2}+m_{3}+\cdots+m_{k-1}} q^{\frac{m_{1}\left(m_{1}+1\right)}{2}+\frac{m_{2}\left(m_{2}+1\right)}{2}+\cdots+\frac{m_{k-1}\left(m_{k-1}+1\right)}{2}} w^{m_{1}} \beta_{m_{k-1}}}{(q)_{m_{1}}(q)_{m_{1}-m_{2}} \cdots(q)_{m_{k-2}-m_{k-1}}} \\
= & \frac{(-w q)_{\infty}}{\left(q^{2}\right)_{\infty}} \sum_{n \geq 0} \frac{\left(-w^{-1} q\right)_{n}(-1)^{k n} q^{\frac{(k-1) n(n+1)}{2}} w^{n} \alpha_{n}}{(-w q)_{n}}
\end{aligned}
$$

where $\left(\alpha_{n}, \beta_{n}\right)$ is any Bailey pair relative to $a=q$, and $w \in \mathbb{C}$. We require a single Bailey pair relative to $a=q$. Specifically this is the Bailey pair $B(3)$ of Slater [30], which is defined by

$$
\alpha_{n}^{B 3}:=\frac{(-1)^{n} q^{\frac{n(3 n+1)}{2}}\left(1-q^{2 n+1}\right)}{(1-q)}, \quad \beta_{n}^{B 3}:=\frac{1}{(q)_{n}} .
$$

The additional $q$-series identity we require are as follows. We have two identities of Euler [19, (II.1) and (II.2)],

$$
\begin{gathered}
\sum_{n \geq 0} \frac{z^{n}}{(q)_{n}}=\frac{1}{(z)_{\infty}}, \\
\sum_{n \geq 0} \frac{(-1)^{n} z^{n} q^{\frac{n(n-1)}{2}}}{(q)_{n}}=(z)_{\infty} .
\end{gathered}
$$

More generally, the $q$-binomial theorem [19, (II.3)] states that

$$
\sum_{n \geq 0} \frac{(a)_{n} z^{n}}{(q)_{n}}=\frac{(a z)_{\infty}}{(z)_{\infty}}
$$

We also need two forms of Heine's transformation [19, (III.1) and (III.2)], which are

$$
\begin{aligned}
& \sum_{n \geq 0} \frac{(a, b)_{n} z^{n}}{(c, q)_{n}}=\frac{(b, a z)_{\infty}}{(c, z)_{\infty}} \sum_{n \geq 0} \frac{\left(\frac{c}{b}, z\right)_{n} b^{n}}{(a z, q)_{n}} \\
& \sum_{n \geq 0} \frac{(a, b)_{n} z^{n}}{(c, q)_{n}}=\frac{\left(\frac{c}{b}, b z\right)_{\infty}}{(c, z)_{\infty}} \sum_{n \geq 0} \frac{\left(\frac{a b z}{c}, b\right)_{n}\left(\frac{c}{b}\right)^{n}}{(b z, q)_{n}} .
\end{aligned}
$$

Lastly, we use Lemma 1 of [4] written as

$$
\frac{1}{\left(\zeta q^{\frac{1}{2}}, \zeta^{-1} q^{\frac{1}{2}}\right)_{\infty}}=\frac{1}{(q)_{\infty}^{2}} \sum_{\substack{n_{1} \in \mathbb{Z} \\ n_{2} \geq\left|n_{1}\right|}}(-1)^{n_{1}+n_{2}} q^{\frac{n_{2}\left(n_{2}+1\right)}{2}-\frac{n_{1}^{2}}{2}} \zeta^{n_{1}}
$$

We note that the summation bound $n_{2} \geq\left|n_{1}\right|$ in (3.8) can be replaced by $n_{2} \geq n_{1}$.

\section{IDENTITIES With HALF-CHARACTERISTIC}

In this section we extend Theorems 1.1 and 1.2 from the introduction to half-characteristic. 
Proposition 4.1. Suppose $k \geq 1$ and $w \in \mathbb{C}$. Then

$$
\begin{aligned}
& \sum_{n_{1}, n_{2}, \ldots, n_{k} \geq 0} \frac{q^{n_{1} n_{2}+n_{2} n_{3}+\cdots+n_{k-1} n_{k}+n_{1}+n_{2}+\cdots+n_{k}}(-w)_{n_{1}}}{(q)_{n_{1}}^{2}(q)_{n_{2}}^{2} \cdots(q)_{n_{k}}^{2}} \\
&=\mathrm{CT}_{\zeta_{1}, \zeta_{2}, \ldots, \zeta_{k}} \frac{\phi\left(-w q^{\frac{1}{2}} \zeta_{1}\right)}{\phi\left(q^{\frac{1}{2}} \zeta_{1}\right)}\left(\prod_{j=2}^{k} \frac{1}{\phi\left(q^{\frac{1}{2}} \zeta_{j}\right) \phi\left(q^{\frac{1}{2}} \zeta_{j-1}^{-1}\right)}\right) \frac{1}{\phi\left(q^{\frac{1}{2}} \zeta_{k}^{-1}\right)},
\end{aligned}
$$

where the $\zeta_{j}$ are non-commuting variables with $\zeta_{j} \zeta_{j+1}=q \zeta_{j+1} \zeta_{j}$ for $1 \leq j \leq k-1$.

Proof: The proof is similar to that of Theorems 1.1 and 1.2. We expand $\phi\left(-w q^{\frac{1}{2}} \zeta_{1}\right) / \phi\left(q^{\frac{1}{2}} \zeta_{1}\right)$ with the $q$-binomial theorem (3.5) and all other products are expanded with Euler's identity (3.3). By doing so we have

$$
\begin{aligned}
& \frac{\phi\left(-w q^{\frac{1}{2}} \zeta_{1}\right)}{\phi\left(q^{\frac{1}{2}} \zeta_{1}\right)}\left(\prod_{j=2}^{k} \frac{1}{\phi\left(q^{\frac{1}{2}} \zeta_{j}\right) \phi\left(q^{\frac{1}{2}} \zeta_{j-1}^{-1}\right)}\right) \frac{1}{\phi\left(q^{\frac{1}{2}} \zeta_{k}^{-1}\right)} \\
& =\sum_{\mathbf{n}, \mathbf{m} \in \mathbb{N}_{0}^{k}} \frac{q^{\frac{n_{1}+m_{1}+n_{2}+m_{2}+\cdots+n_{k}+m_{k}}{2}}(-w)_{n_{1}}}{(q)_{n_{1}}(q)_{m_{1}}(q)_{n_{2}}(q)_{m_{2}} \cdots(q)_{n_{k}}(q)_{m_{k}}} \zeta_{1}^{n_{1}}\left(\prod_{j=2}^{k} \zeta_{j}^{n_{j}} \zeta_{j-1}^{-m_{j-1}}\right) \zeta_{k}^{-m_{k}} \\
& =\sum_{\mathbf{n}, \mathbf{m} \in \mathbb{N}_{0}^{k}} \frac{q^{\frac{n_{1}+m_{1}+n_{2}+m_{2}+\cdots+n_{k}+m_{k}}{2}+n_{2} m_{1}+n_{3} m_{2}+\cdots+n_{k} m_{k-1}(-w)_{n_{1}} \zeta_{1}^{n_{1}-m_{1}} \zeta_{2}^{n_{2}-m_{2}} \cdots \zeta_{k}^{n_{k}-m_{k}}}}{(q)_{n_{1}}(q)_{m_{1}}(q)_{n_{2}}(q)_{m_{2}} \cdots(q)_{n_{k}}(q)_{m_{k}}} .
\end{aligned}
$$

The constant term then clearly comes from taking $m_{j}=n_{j}$ and the proposition follows.

In the lemma below, we give an intermediate identity that is required so that we may apply Bailey's lemma.

Lemma 4.2. Suppose $k \geq 2$ and $w \in \mathbb{C}$. Then

$$
\begin{aligned}
& \sum_{n_{1}, n_{2}, \ldots, n_{k} \geq 0} \frac{q^{n_{1} n_{2}+n_{2} n_{3}+\cdots+n_{k-1} n_{k}+n_{1}+n_{2}+\cdots+n_{k}}(-w)_{n_{1}}}{(q)_{n_{1}}^{2}(q)_{n_{2}}^{2} \cdots(q)_{n_{k}}^{2}} \\
= & \frac{1}{(q)_{\infty}^{k}} \sum_{m_{1}, m_{2}, \ldots, m_{k-1} \geq 0} \frac{(-1)^{m_{2}+\cdots+m_{k-1}} q^{\frac{m_{1}\left(m_{1}+1\right)}{2}+\frac{m_{2}\left(m_{2}+1\right)}{2}+\cdots+\frac{m_{k-1}\left(m_{k-1}+1\right)}{2}} w^{m_{1}}\left(-w^{-1} q\right)_{m_{1}}}{(q)_{m_{1}}(q)_{m_{1}-m_{2}}(q)_{m_{2}-m_{3}} \cdots(q)_{m_{k-2}-m_{k-1}}} .
\end{aligned}
$$

Proof: We begin by reevaluating the constant term in (4.1) by applying (2.2) and expanding the products with (3.5), (3.3), and (3.8). For convenience with the indices, we instead use 
$\zeta_{0}, \zeta_{1}, \ldots, \zeta_{k-1}$. With this all mind, we find that

$$
\begin{aligned}
& \frac{\phi\left(-w q^{\frac{1}{2}} \zeta_{0}\right)}{\phi\left(q^{\frac{1}{2}} \zeta_{0}\right)}\left(\prod_{j=1}^{k-1} \frac{1}{\phi\left(q^{\frac{1}{2}} \zeta_{j}\right) \phi\left(q^{\frac{1}{2}} \zeta_{j-1}^{-1}\right)}\right) \frac{1}{\phi\left(q^{\frac{1}{2}} \zeta_{k-1}^{-1}\right)} \\
& =\frac{\phi\left(-w q^{\frac{1}{2}} \zeta_{0}\right)}{\phi\left(q^{\frac{1}{2}} \zeta_{0}\right) \phi\left(q^{\frac{1}{2}} \zeta_{0}^{-1}\right)} \prod_{j=1}^{k-1} \frac{1}{\phi\left(-\zeta_{j} \zeta_{j-1}^{-1}\right) \phi\left(q^{\frac{1}{2}} \zeta_{j}\right) \phi\left(q^{\frac{1}{2}} \zeta_{j}^{-1}\right)}
\end{aligned}
$$

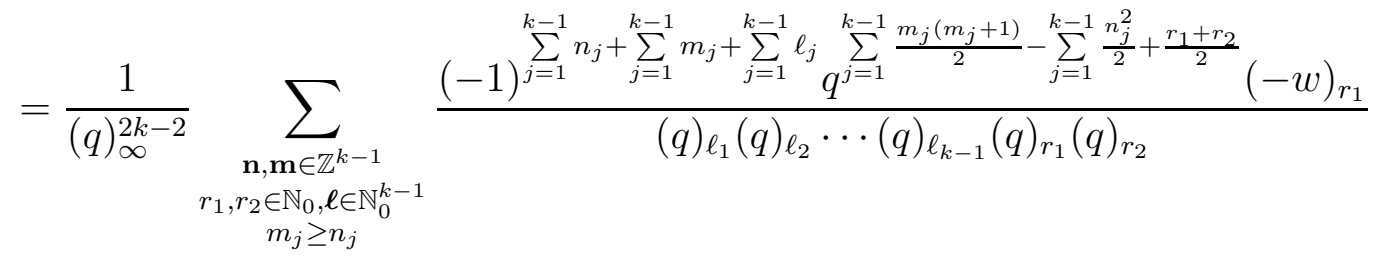

$$
\begin{aligned}
& \times \zeta_{0}^{r_{1}-r_{2}} \prod_{j=1}^{k-1}\left(\zeta_{j} \zeta_{j-1}^{-1}\right)^{\ell_{j}} \zeta_{j}^{n_{j}} \\
& =\frac{1}{(q)_{\infty}^{2 k-2}} \sum_{\substack{\mathbf{n}, \mathbf{m} \in \mathbb{Z}^{k-1} \\
r_{1}, r_{2} \in \mathbb{N}_{0}, \ell \in \mathbb{N}_{0}^{k-1} \\
m_{j} \geq n_{j}}} \frac{(-1)^{\sum_{j=1}^{k-1} n_{j}+\sum_{j=1}^{k-1} m_{j}+\sum_{j=1}^{k-1} \ell_{j}} \sum^{j=1} \frac{\sum_{j} \frac{m_{j}\left(m_{j}+1\right)}{2}-\sum_{j=1}^{k-1} \frac{n_{j}^{2}}{2}+\sum_{j=1}^{k-1} \frac{\ell_{j}\left(\ell_{j}+1\right)}{2}+\frac{r_{1}+r_{2}}{2}}{2}(-w)_{r_{1}}}{(q)_{\ell_{1}}(q)_{\ell_{2}} \cdots(q)_{\ell_{k-1}}(q)_{r_{1}}(q)_{r_{2}}} \\
& \times \zeta_{0}^{r_{1}-r_{2}-\ell_{1}}\left(\prod_{j=1}^{k-2} \zeta_{j}^{n_{j}+\ell_{j}-\ell_{j+1}}\right) \zeta_{k-1}^{n_{k}+\ell_{k-1}}
\end{aligned}
$$

The constant term comes from $n_{j}=\ell_{j+1}-\ell_{j}$ for $1 \leq j \leq k-2, n_{k-1}=-\ell_{k-1}$, and $r_{2}=r_{1}-\ell_{1}$. For the index bounds, we replace $m_{k-1} \geq n_{k-1}$ with $m_{k-1} \geq\left|n_{k-1}\right|$. Thus by Proposition 4.1 ,

$$
\begin{aligned}
& \sum_{n_{1}, n_{2}, \ldots, n_{k} \geq 0} \frac{q^{n_{1} n_{2}+n_{2} n_{3}+\cdots+n_{k-1} n_{k}+n_{1}+n_{2}+\cdots+n_{k}}(-w)_{n_{1}}}{(q)_{n_{1}}^{2}(q)_{n_{2}}^{2} \cdots(q)_{n_{k}}^{2}} \\
& =\frac{1}{(q)_{\infty}^{2 k-2}} \sum_{\substack{\mathbf{m} \in \mathbb{Z}^{k-1}, \ell \in \mathbb{N}_{0}^{k-1} \\
r \in \mathbb{N}_{0}, r \geq \ell_{1} \\
m_{k-1} \geq \ell_{k-1} \\
m_{j} \geq \ell_{j+1}-\ell_{j}}} \frac{(-1)^{\sum_{j=1}^{k-1} m_{j}+\sum_{j=2}^{k-2} \ell_{j} \sum^{j=1} \frac{k-1}{m_{j}\left(m_{j}+1\right)}+\frac{\ell_{1}^{2}}{2}+\sum_{j=2}^{k-1} \frac{\ell_{j}\left(\ell_{j}+1\right)}{2}-\sum_{j=1}^{k-2} \frac{\left(\ell_{j+1}-\ell_{j}\right)^{2}}{2}-\frac{\ell_{k-1}^{2}}{2}+r}(-w)_{r}}{(q)_{\ell_{1}}(q)_{\ell_{2}} \cdots(q)_{\ell_{k-1}}(q)_{r}(q)_{r-\ell_{1}}} \\
& =\frac{1}{(q)_{\infty}^{2 k-2}} \sum_{r \in \mathbb{N}_{0}, \ell, \mathbf{m} \in \mathbb{N}_{0}^{k-1}} \frac{(-1)^{\sum_{j=1}^{k-1} m_{j}+\sum_{j=1}^{k-1} \ell_{j}} q^{\sum_{j=1}^{k-1} \frac{m_{j}\left(m_{j}+1\right)}{2}+\sum_{j=1}^{k-2} \frac{\ell_{j}\left(\ell_{j}+1\right)}{2}+\frac{\ell_{k-1}\left(\ell_{k-1}+3\right)}{2}}(-w)_{r+\ell_{1}}}{(q)_{\ell_{1}}(q)_{\ell_{2}} \cdots(q)_{\ell_{k-1}}(q)_{r}(q)_{r+\ell_{1}}} \\
& \times q^{-\ell_{1} m_{1}+\sum_{j=2}^{k-2} \ell_{j}\left(m_{j-1}-m_{j}\right)+\ell_{k-1}\left(m_{k-2}+m_{k-1}\right)+r} .
\end{aligned}
$$


Due to convergence issues in certain calculations below, we view the far right-hand side of (4.2) as the $x \rightarrow 1$ case of

$$
\begin{aligned}
F(x):= & \frac{1}{(q)_{\infty}^{2 k-2}} \sum_{r \in \mathbb{N}_{0}, \ell, \mathbf{m} \in \mathbb{N}_{0}^{k-1}} \frac{(-1)^{\sum_{j=1}^{k-1} m_{j}+\sum_{j=1}^{k-1} \ell_{j} \sum^{k=1} \frac{m_{j}\left(m_{j}+1\right)}{2}+\sum_{j=1}^{k-2} \frac{\ell_{j}\left(\ell_{j}+1\right)}{2}+\frac{\ell_{k-1}\left(\ell_{k-1}+3\right)}{2}}(-x w)_{r+\ell_{1}}}{(q)_{\ell_{1}}(q)_{\ell_{2}} \cdots(q)_{\ell_{k-1}}(q)_{r}(x q)_{r+\ell_{1}}} \\
& \times q^{-\ell_{1} m_{1}+\sum_{j=2}^{k-2} \ell_{j}\left(m_{j-1}-m_{j}\right)+\ell_{k-1}\left(m_{k-2}+m_{k-1}\right)+r} .
\end{aligned}
$$

We transform the inner sum on $r$ with Heine's transformation (3.6) with $a=0, b=-x w q^{\ell_{1}}$, $c=x q^{\ell_{1}+1}$, and $z=q$, as

$$
\sum_{r \geq 0} \frac{(-x w)_{r+\ell_{1}} q^{r}}{(q)_{r}(x q)_{r+\ell_{1}}}=\frac{(-x w)_{\ell_{1}}}{(x q)_{\ell_{1}}} \sum_{r \geq 0} \frac{\left(-x w q^{\ell_{1}}\right)_{r} q^{r}}{(q)_{r}\left(x q^{\ell_{1}+1}\right)_{r}}=\frac{(-x w)_{\infty}}{(x q)_{\infty}(q)_{\infty}} \sum_{r \geq 0}\left(-w^{-1} q\right)_{r}(-1)^{r} x^{r} w^{r} q^{\ell_{1} r}
$$

we note that when $x=1$, the final series above is not absolutely convergent for all $w$ and $\ell_{1}$. Thus for $|x w|<1$,

$$
\begin{aligned}
F(x)= & \frac{(-x w)_{\infty}}{(x q)_{\infty}(q)_{\infty}^{2 k-1}} \sum_{r \in \mathbb{N}_{0}, \ell, \mathbf{m} \in \mathbb{N}_{0}^{k-1}} \frac{(-1)^{\sum_{j=1}^{k-1} m_{j}+\sum_{j=1}^{k-1} \ell_{j}+r}{q^{j=1}}^{k-1} \frac{m_{j}\left(m_{j}+1\right)}{2}+\sum_{j=1}^{k-2} \frac{\ell_{j}\left(\ell_{j}+1\right)}{2}+\frac{\ell_{k-1}\left(\ell_{k-1}+3\right)}{2}}{(q)_{\ell_{1}}(q)_{\ell_{2}} \cdots(q)_{\ell_{k-1}}} \\
& \times q^{-\ell_{1} m_{1}+\sum_{j=2}^{k-2} \ell_{j}\left(m_{j-1}-m_{j}\right)+\ell_{k-1}\left(m_{k-2}+m_{k-1}\right)+\ell_{1} r} x^{r} w^{r}\left(-w^{-1} q\right)_{r} .
\end{aligned}
$$

We evaluate the inner sums on each $\ell_{j}$ with (3.4) to find that

$$
\begin{gathered}
\sum_{\ell_{1} \geq 0} \frac{(-1)^{\ell_{1}} q^{\ell_{1}\left(1+r-m_{1}\right)} q^{\frac{\ell_{1}\left(\ell_{1}-1\right)}{2}}}{(q)_{\ell_{1}}}=\left(q^{1+r-m_{1}}\right)_{\infty}=\frac{(q)_{\infty}}{(q)_{r-m_{1}}}, \\
\sum_{\ell_{j} \geq 0} \frac{(-1)^{\ell_{j}} q^{\ell_{j}\left(1+m_{j-1}-m_{j}\right)} q^{\frac{\ell_{j}\left(\ell_{j}-1\right)}{2}}}{(q)_{\ell_{j}}}=\left(q^{1+m_{j-1}-m_{j}}\right)=\frac{(q)_{\infty}}{(q)_{m_{j-1}-m_{j}}}, \quad \text { for } 2 \leq j \leq k-2, \\
\sum_{\ell_{k-1} \geq 0} \frac{(-1)^{\ell_{k-1}} q^{\ell_{j}\left(2+m_{k-2}+m_{k-1}\right)} q^{\frac{\ell_{k-1}\left(\ell_{k-1}-1\right)}{2}}}{(q)_{\ell_{k-1}}}=\left(q^{2+m_{k-2}+m_{k-1}}\right)=\frac{(q)_{\infty}}{(q)_{m_{k-2}+m_{k-1}+1}} .
\end{gathered}
$$

Thus, for $|x w|<1$,

$$
\begin{aligned}
F(x) & =\frac{(-x w)_{\infty}}{(x q)_{\infty}(q)_{\infty}^{k}} \sum_{r, m_{1}, m_{2}, \ldots, m_{k-1} \geq 0} \frac{(-1)^{\sum_{j=1}^{k-1} m_{j}+r \sum^{\sum_{j=1}^{k-1} \frac{m_{j}\left(m_{j}+1\right)}{2}} x^{r} w^{r}\left(-w^{-1} q\right)_{r}}}{(q)_{r-m_{1}}(q)_{m_{1}-m_{2}}(q)_{m_{2}-m_{3}} \cdots(q)_{m_{k-3}-m_{k-2}}(q)_{m_{k-2}+m_{k-1}+1}} \\
& =\frac{(-x w)_{\infty}}{(x q)_{\infty}(q)_{\infty}^{k}} \sum_{r, m_{1}, m_{2}, \ldots, m_{k-1} \geq 0} \frac{(-1)^{\sum_{j=1}^{k-1} m_{j}+r} q^{\sum_{j=1}^{k-1} \frac{m_{j}\left(m_{j}+1\right)}{2}} x^{r} w^{r}\left(-w^{-1} q\right)_{r}}{(q)_{r-m_{1}}(q)_{m_{1}-m_{2}}(q)_{m_{2}-m_{3}} \cdots(q)_{m_{k-2}-m_{k-1}}},
\end{aligned}
$$


where the second equality follows from Heine's transformation (3.7) with $a \rightarrow \infty, b=q$, $c=q^{2+m_{k-2}}, z=\frac{q}{a}$, applied to the inner sum on $m_{k-1}$. By (3.5), the sum on $r$ is

$$
\begin{aligned}
\sum_{r \geq 0} \frac{(-1)^{r} x^{r} w^{r}\left(-w^{-1} q\right)_{r}}{(q)_{r-m_{1}}} & =(-1)^{m_{1}} x^{m_{1}} w^{m_{1}}\left(-w^{-1} q\right)_{m_{1}} \sum_{r \geq 0} \frac{(-1)^{r} x^{r} w^{r}\left(-w^{-1} q^{m_{1}+1}\right)_{r}}{(q)_{r}} \\
& =\frac{(-1)^{m_{1}} x^{m_{1}} w^{m_{1}}\left(-w^{-1} q\right)_{m_{1}}(x q)_{\infty}}{(x q)_{m_{1}}(-x w)_{\infty}}
\end{aligned}
$$

and so

$$
F(x)=\frac{1}{(q)_{\infty}^{k}} \sum_{m_{1}, m_{2}, \ldots, m_{k-1} \geq 0} \frac{(-1)^{\sum_{j=2}^{k-1} m_{j}} q^{\sum_{j=1}^{k-1} \frac{m_{j}\left(m_{j}+1\right)}{2}} x^{m_{1}} w^{m_{1}}\left(-w^{-1} q\right)_{m_{1}}}{(x q)_{m_{1}}(q)_{m_{1}-m_{2}}(q)_{m_{2}-m_{3}} \cdots(q)_{m_{k-2}-m_{k-1}}} .
$$

This form of $F(x)$ is well defined for exactly the same values of $x$ as (4.3) and so we find the lemma follows by setting $x=1$.

Our extension of Theorems 1.1 and 1.2 to the series in (1.3) is given here.

Theorem 4.3. Suppose $k \geq 2$. Then

$$
\begin{gathered}
\sum_{n_{1}, n_{2}, \ldots, n_{k} \geq 0} \frac{q^{n_{1} n_{2}+n_{2} n_{3}+\cdots+n_{k-1} n_{k}+n_{1}+n_{2}+\cdots+n_{k}}(-1)_{n_{1}}}{(q)_{n_{1}}^{2}(q)_{n_{2}}^{2} \cdots(q)_{n_{k}}^{2}} \\
=\frac{(-q)_{\infty}}{(q)_{\infty}^{k+1}}\left(\sum_{n \geq 0}+(-1)^{k} \sum_{n<0}\right)(-1)^{(k+1) n} q^{\frac{(k+2) n^{2}+k n}{2}}, \\
\sum_{n_{1}, n_{2}, \ldots, n_{k} \geq 0} \frac{q^{n_{1} n_{2}+n_{2} n_{3}+\cdots+n_{k-1} n_{k}+n_{1}+n_{2}+\cdots+n_{k}}\left(-q^{\frac{1}{2}}\right)_{n_{1}}}{(q)_{n_{1}}^{2}(q)_{n_{2}}^{2} \cdots(q)_{n_{k}}^{2}} \\
=\frac{\left(-q^{\frac{1}{2}}\right)_{\infty}}{(q)_{\infty}^{k+1}}\left(\sum_{n \geq 0}+(-1)^{k} \sum_{n<0}\right)(-1)^{(k+1) n} q^{\frac{(k+2) n^{2}+(k+1) n}{2}} .
\end{gathered}
$$

Proof: We see that the series in the right-hand side of the identity in Lemma 4.2 perfectly matches the statement of Bailey's lemma (3.1) with the Bailey pair in (3.2). By combining these statements we have that

$$
\begin{gathered}
\sum_{n_{1}, n_{2}, \ldots, n_{k} \geq 0} \frac{q^{n_{1} n_{2}+n_{2} n_{3}+\cdots+n_{k-1} n_{k}+n_{1}+n_{2}+\cdots+n_{k}}(-w)_{n_{1}}}{(q)_{n_{1}}^{2}(q)_{n_{2}}^{2} \cdots(q)_{n_{k}}^{2}} \\
=\frac{(-w q)_{\infty}}{(q)_{\infty}^{k+1}} \sum_{n \geq 0} \frac{\left(-w^{-1} q\right)_{n}(-1)^{(k+1) n} q^{\frac{(k+2) n^{2}+k n}{2}} w^{n}\left(1-q^{2 n+1}\right)}{(-w q)_{n}} .
\end{gathered}
$$

When $w=1$, the right-hand side of (4.4) simplifies to

$$
\begin{aligned}
\frac{(-q)_{\infty}}{(q)_{\infty}^{k+1}} \sum_{n \geq 0}(-1)^{(k+1) n} q^{\frac{(k+2) n^{2}+k n}{2}}(1 & \left.-q^{2 n+1}\right) \\
& =\frac{(-q)_{\infty}}{(q)_{\infty}^{k+1}}\left(\sum_{n \geq 0}+(-1)^{k} \sum_{n<0}\right)(-1)^{(k+1) n} q^{\frac{(k+2) n^{2}+k n}{2}},
\end{aligned}
$$


as claimed. When $w=q^{\frac{1}{2}}$, the right-hand side of (4.4) instead simplifies as

$$
\begin{aligned}
\frac{\left(-q^{\frac{1}{2}}\right)_{\infty}}{(q)_{\infty}^{k+1}} \sum_{n \geq 0}(-1)^{(k+1) n} q^{\frac{(k+2) n^{2}+(k+1) n}{2}} & \left(1-q^{n+\frac{1}{2}}\right) \\
= & \frac{\left(-q^{\frac{1}{2}}\right)_{\infty}}{(q)_{\infty}^{k+1}}\left(\sum_{n \geq 0}+(-1)^{k} \sum_{n<0}\right)(-1)^{(k+1) n} q^{\frac{(k+2) n^{2}+(k+1) n}{2}} .
\end{aligned}
$$

There is a similar identity that comes from taking $w=-q^{\frac{1}{2}}$. We state this identity in the proposition below, but omit the proof as it is essentially the same as the other two cases.

Proposition 4.4. Suppose $k \geq 2$. Then

$$
\begin{aligned}
\sum_{n_{1}, n_{2}, \ldots, n_{k} \geq 0} \frac{q^{n_{1} n_{2}+n_{2} n_{3}+\cdots+n_{k-1} n_{k}+n_{1}+n_{2}+\cdots+n_{k}}\left(q^{\frac{1}{2}}\right)_{n_{1}}}{(q)_{n_{1}}^{2}(q)_{n_{2}}^{2} \cdots(q)_{n_{k}}^{2}} & \\
& =\frac{\left(q^{\frac{1}{2}}\right)_{\infty}}{(q)_{\infty}^{k+1}}\left(\sum_{n \geq 0}+(-1)^{k} \sum_{n<0}\right)(-1)^{k n} q^{\frac{(k+2) n^{2}+(k+1) n}{2}} .
\end{aligned}
$$

\section{IDENTITIES FOR CHARACTERS}

In this section we study $q$-series identities coming from the formal inversion $q \mapsto q^{-1}$ in the $q$-hypergeometric term in (1.1) and (1.2). As in [21] we make use of quantum dilogarithms to prove the following result. The same result was discussed in [14].

Proposition 5.1. Let

$$
\operatorname{ch}_{W}(\tau)=\sum_{n_{1}, n_{2}, \ldots, n_{2 k-1} \geq 0} \frac{q^{\sum_{i=1}^{2 k-1} n_{i}^{2}-\sum_{i=1}^{2 k-2} n_{i} n_{i+1}}}{(q)_{n_{1}}(q)_{n_{2}} \cdots(q)_{n_{2 k-1}}}
$$

denote the character of the principal subspaces of the level one vertex operator algebra $W\left(\Lambda_{0}\right)$ of type $A_{2 k-1}^{(1)}$ [13, 17]. Then

$$
\sum_{n_{1}, n_{2}, \ldots, n_{2 k} \geq 0} \frac{q^{\sum_{i=1}^{2 k} n_{i}^{2}-\sum_{i=1}^{2 k-1} n_{i} n_{i+1}}}{(q)_{n_{1}}^{2}(q)_{n}^{2} \cdots(q)_{n_{2 k}}^{2}}=\frac{1}{(q)_{\infty}^{2 k}} \operatorname{ch}_{W}(\tau)
$$

Moreover, after multiplication by $q^{a}$ for some $a \in \mathbb{Q}$, this a modular form. 
Proof: This follows by verifying that

$$
\begin{aligned}
& \sum_{n_{1}, \ldots, n_{k} \geq 0} \frac{q^{n_{1}^{2}+n_{2}^{2}+\cdots+n_{k}^{2}-n_{1} n_{2}-n_{2} n_{3}-\cdots-n_{k-1} n_{k}}}{(q)_{n_{1}}^{2}(q)_{n_{2}}^{2} \cdots(q)_{n_{k}}^{2}} \\
= & \mathrm{CT}_{\zeta_{1}, \zeta_{2}, \ldots, \zeta_{k}} \phi\left(q^{\frac{1}{2}} \zeta_{1}\right)\left(\prod_{j=2}^{k} \phi\left(q^{\frac{1}{2}} \zeta_{j}\right) \phi\left(q^{\frac{1}{2}} \zeta_{j-1}^{-1}\right)\right) \phi\left(q^{\frac{1}{2}} \zeta_{k}^{-1}\right) \\
= & \mathrm{CT}_{\zeta_{1}, \zeta_{2}, \ldots, \zeta_{k}} \phi\left(q^{\frac{1}{2}} \zeta_{1}\right) \phi\left(q^{\frac{1}{2}} \zeta_{1}^{-1}\right) \prod_{j=2}^{k} \phi\left(-q \zeta_{j} \zeta_{j-1}^{-1}\right) \phi\left(q^{\frac{1}{2}} \zeta_{j}\right) \phi\left(q^{\frac{1}{2}} \zeta_{j}^{-1}\right) \\
= & \frac{1}{(q)_{\infty}^{k}} \sum_{n_{1}, \ldots, n_{k-1} \geq 0} \frac{q^{n_{1}^{2}+n_{2}^{2}+\cdots+n_{k-1}^{2}-n_{1} n_{2}-n_{2} n_{3}-\cdots-n_{k-2} n_{k-1}}}{(q)_{n_{1}}(q)_{n_{2}} \cdots(q)_{n_{k-1}}}
\end{aligned}
$$

where the $\zeta_{i}$ are non-commuting variables with $\zeta_{i} \zeta_{i+1}=q^{-1} \zeta_{i+1} \zeta_{i}$.

For the modularity, we first use an identity from [32, Theorem 2.1]. This allows us to write $\operatorname{ch}_{W}(\tau)$ as a modular Wronskian of certain theta functions [12] (as usual, we have to multiply by $q^{a}$ ), which is known to be modular with respect to some congruence subgroup 25 .

For the $q$-hypergeometric series appearing in Theorem 1.1 we expect a family of modular identities. We first define $F_{k}(q)$ by letting

$$
\sum_{n_{1}, n_{2}, \ldots, n_{2 k+1} \geq 0} \frac{q^{\sum_{i=1}^{2 k+1} n_{i}^{2}-\sum_{i=1}^{2 k} n_{i} n_{i+1}}}{(q)_{n_{1}}^{2}(q)_{n_{2}}^{2} \cdots(q)_{n_{2 k+1}}^{2}}=\frac{1}{(q)_{\infty}^{2 k+1}} F_{k}(q) .
$$

We believe the following to be true.

Conjecture 5.2. For $k \geq 1$, we have

$$
F_{k}(q)=\operatorname{ch}\left[L_{\mathfrak{s p}(2 k)}\left(\Lambda_{0}\right)\right](q),
$$

which is the character of the (suitably normalized) level one affine vertex algebra $L_{\mathfrak{s p}(2 k)}\left(\Lambda_{0}\right)$ of type $C_{k}^{(1)}$.

Now we provide evidence in support of Conjecture (5.2).

Proposition 5.3. For $k \geq 1$, we have

$$
F_{k}(q)=1+(2 k+1) k q+\frac{1}{3} k\left(3+5 k+4 k^{3}\right) q^{2}+O\left(q^{3}\right) .
$$

Proof: We first let

$$
\sum_{n_{1}, \ldots, n_{2 k+1} \geq 0} \frac{q^{\sum_{i=1}^{2 k+1} n_{i}^{2}-\sum_{i=1}^{2 k} n_{i} n_{i+1}}}{(q)_{n_{1}}^{2}(q)_{n}^{2} \cdots(q)_{n_{2 k+1}}^{2}}=1+d_{k} q+a_{k} q^{2}+O\left(q^{3}\right) .
$$

The linear term is clear, it simply counts the number of positive roots in the root system of type $A_{2 k+1}$. Put differently, it counts the number of positive integral solutions of

$$
\sum_{i=1}^{2 k+1} n_{i}^{2}-\sum_{i=1}^{2 k} n_{i} n_{i+1}=1
$$


These solutions are exactly of the form $\left(n_{1}, \ldots, n_{2 k+1}\right)=(0, . ., 0,1, \ldots, 1,0, . ., 0)$, and it is easily seen that the number of them is $d_{k}=(2 k+1)(k+1)$.

We claim that $a_{k}=\frac{1}{3}(1+k)(1+2 k)\left(6+3 k+2 k^{2}\right)$. For this, we first note that the contribution from the quadratic term comes from two sources, specifically from the terms $\frac{q}{(q)_{n_{1}}^{2} \cdots(q)_{n_{2 k+1}}^{2}}$ and $\frac{q^{2}}{(q)_{n_{1}}^{2} \cdots(q)_{n_{2 k+1}}^{2}}$. As such we have to analyze non-negative integral solutions of (5.2) and of

$$
\sum_{i=1}^{2 k+1} n_{i}^{2}-\sum_{i=1}^{2 k} n_{i} n_{i+1}=2
$$

Clearly the solutions of (15.2) contribute a total of $\sum_{0 \leq j<i \leq 2 k+1} 2(i-j)=\frac{2}{3}(k+1)(1+2 k)(3+$ $2 k$ ) to the quadratic coefficient. For the second equation, no solution has $n_{i} \geq 3$, and so we may assume $0 \leq n_{i} \leq 2$. Next we consider two types of solutions: (a) solutions with all $n_{i} \leq 1$, and (b) solutions with at least one $n_{i}=2$. In case (a), we see that in each such solution there must be exactly two substrings of 1s:

$$
(0, \ldots, 0,1, \ldots, 1,0, \ldots, 0,1, \ldots, 1,0, \ldots, 0) .
$$

For (b), each solution takes form:

$$
(0, . ., 0,1, \ldots 1,2, \ldots, 2,1, \ldots, 1,0, . ., 0) .
$$

In both cases the initial or terminal subsequence of 0s can be empty. For either (a) and (b), it is easy to see combinatorially that we have precisely $\left(\begin{array}{c}(2 k+1)+1 \\ 4\end{array}\right)$ solutions. Therefore, altogether there are

$$
2\left(\begin{array}{c}
2 k+2 \\
4
\end{array}\right)+\frac{2}{3}(k+1)(1+2 k)(3+2 k)=\frac{1}{3}(1+k)(1+2 k)\left(6+3 k+2 k^{2}\right)
$$

solutions. Next we note $\frac{1}{(q)_{\infty}^{2 k+1}}=1+(2 k+1) q+\left(2+5 k+2 k^{2}\right) q^{2}+O\left(q^{3}\right)$ and we write

$$
\sum_{n_{1}, n_{2}, \ldots, n_{2 k+1} \geq 0} \frac{q^{\sum_{i=1}^{2 k+1} n_{i}^{2}-\sum_{i=1}^{2 k} n_{i} n_{i+1}}}{(q)_{n_{1}}^{2}(q)_{n}^{2} \cdots(q)_{n_{2 k+1}}^{2}}=\left(1+(2 k+1) q+\left(2+5 k+2 k^{2}\right) q^{2}+\cdots\right) F_{k}(q) .
$$

Expanding $F(q)=1+a q+b q^{2}+O\left(q^{3}\right)$ and and solving for $a$ and $b$ gives $a=(2 k+1) k$ and $b=\frac{1}{3}\left(3 k+5 k^{2}+4 k^{4}\right)$ as claimed.

Since this is in agreement with the known properties of $\operatorname{ch}\left[L_{\mathfrak{s p}(2 k)}\left(\Lambda_{0}\right)\right](\tau)$ (see [29]), our conjecture is valid $O\left(q^{3}\right)$.

Remark. After this work was completed, we became aware of a paper by A.V. Stoyanovsky [31] which identifies the character of the principal subspace for $A_{2 k}^{(1)}$ at level one and the character of $L_{\mathfrak{s p}(2 k)}\left(\Lambda_{0}\right)$. This result together with the main identity in the proof of Proposition 5.1 implies our Conjecture 5.2. Stoyanovsky's identity was also discussed in [11] in connection to Hall-Littellwood polynomials.

\section{IDENTITIES FOR NAHM-TYPE SUMS With HIGHER ORDER POLES}

Our interest is identities for $q$-hypergeometric multi-sums of the form

$$
F\left(r_{1}, r_{2}, \ldots, r_{k}\right):=\sum_{n_{1}, n_{2}, \ldots, n_{k} \geq 0} \frac{q^{n_{1}+n_{2}+\cdots+n_{k}+n_{1} n_{2}+n_{2} n_{3}+\cdots+n_{k-1} n_{k}}}{(q)_{n_{1}}^{r_{1}}(q)_{n_{2}}^{r_{2}} \cdots(q)_{n_{k}}^{r_{k}}}
$$


where each $r_{i} \geq 1$ and $r_{1}+r_{2}+\cdots+r_{k} \leq 2 k$. While these sums are too general for us to form a single coherent conjecture, we do see a large number of identities for small $k$. In particular, all of the following are either known or easy to prove:

Proposition 6.1. We have,

$$
\begin{aligned}
F(1,1) & =\frac{1}{(1-q)(q)_{\infty}}, \quad F(1,2)=\frac{1}{(q)_{\infty}^{2}}, \quad F(1,3)=\frac{\sum_{n \in \mathbb{Z}} \operatorname{sgn}(n) q^{2 n^{2}+n}}{(q)_{\infty}^{3}}, \\
F(2,2) & =\frac{\left(q, q^{4}, q^{5} ; q^{5}\right)}{(q)_{\infty}^{3}}, \quad F(1,1,1)=\frac{q^{-1}\left(1-(q ; q)_{\infty}\right)}{(q)_{\infty}^{2}}, \quad F(1,1,2)=\frac{\sum_{n \geq 0}(-1)^{n} q^{n(n+3) / 2}}{(q)_{\infty}^{3}} \\
F(1,2,1) & =\frac{1}{(1-q)(q)_{\infty}^{2}}, \quad F(1,2,2)=\frac{1}{(q)_{\infty}^{3}}, \quad F(1,3,1)=\frac{1}{(q)_{\infty}^{3}}, \\
F(1,2,3) & =\frac{\sum_{n \in \mathbb{Z}} \operatorname{sgn}(n) q^{2 n^{2}+n}}{(q)_{\infty}^{4}}, F(1,3,2)=\frac{\left(q, q^{4}, q^{5} ; q^{5}\right)}{(q)_{\infty}^{4}}, F(1,4,1)=\frac{\sum_{n \in \mathbb{Z}} \operatorname{sgn}(n) q^{2 n^{2}+n}}{(q)_{\infty}^{4}} \\
F(2,1,2) & =\frac{1+2 \sum_{n \geq 1}(-1)^{n} q^{\frac{n(n+1)}{2}}}{(1-q)(q)_{\infty}^{4}}, \quad F(2,2,2)=\frac{\sum_{n \in \mathbb{Z}} \operatorname{sgn}(n) q^{3 n^{2}+2 n}}{(q)_{\infty}^{4}}
\end{aligned}
$$

Proof: By (3.3), the following two identities hold,

$$
F(1,1)=\frac{1}{(1-q)(q)_{\infty}}, \quad F(1,2)=\frac{1}{(q)_{\infty}^{2}}
$$

The identity for $F(2,2)$ is simply the $k=1$ case of Theorem 1.2 , For $F(1,3)$, we begin with (3.3) and find that

$$
F(1,3)=\frac{1}{(q)_{\infty}} \sum_{n \geq 0} \frac{q^{n}}{(q)_{n}^{2}}=\frac{1}{(q)_{\infty}^{3}} \sum_{n \geq 0}(-1)^{n} q^{\frac{n(n+1)}{2}}=\frac{1}{(q)_{\infty}^{3}} \sum_{n \in \mathbb{Z}} \operatorname{sgn}(n) q^{n(2 n+1)}
$$

where the second equality follows from (3.6) with $a=b=0$ and $c=z=q$.

Additional usage of (3.3) then yields

$$
\begin{array}{ll}
F(1,2,1)=\frac{F(1,1)}{(q)_{\infty}}=\frac{1}{(1-q)(q)_{\infty}^{2}}, & F(1,2,2)=\frac{F(1,2)}{(q)_{\infty}}=\frac{1}{(q)_{\infty}^{3}}, \\
F(1,3,1)=\frac{F(1,2)}{(q)_{\infty}}=\frac{1}{(q)_{\infty}^{3}}, & F(1,2,3)=\frac{F(1,3)}{(q)_{\infty}}=\frac{1}{(q)_{\infty}^{4}} \sum_{n \in \mathbb{Z}} \operatorname{sgn}(n) q^{2 n^{2}+n} \\
F(1,3,2)=\frac{F(2,2)}{(q)_{\infty}}=\frac{\left(q, q^{4}, q^{5} ; q^{5}\right)_{\infty}}{(q)_{\infty}^{4}}, & F(1,4,1)=\frac{F(3,1)}{(q)_{\infty}}=\frac{1}{(q)_{\infty}^{4}} \sum_{n \in \mathbb{Z}} \operatorname{sgn}(n) q^{2 n^{2}+n}
\end{array}
$$

The identity for $F(2,2,2)$ is Theorem 1.1 with $k=2$. For $F(1,1,1)$, we begin with two applications of (3.3) and then apply (3.6) with $a=b=z=q$ and $c=0$, which is

$$
F(1,1,1)=\frac{1}{(q)_{\infty}^{2}} \sum_{n \geq 0}(q)_{n} q^{n}=\frac{\left(q^{2}\right)_{\infty}}{(q)_{\infty}^{2}} \sum_{n \geq 0} \frac{q^{n}}{\left(q^{2}\right)_{n}}=\frac{1}{(q)_{\infty}} \sum_{n \geq 1} \frac{q^{n-1}}{(q)_{n}}=\frac{q^{-1}}{(q)_{\infty}}\left(\frac{1}{(q)_{\infty}}-1\right)
$$


For $F(1,1,2)$, we begin with (3.3) and end with (3.6),

$$
\begin{aligned}
F(1,1,2) & =\frac{1}{(q)_{\infty}} \sum_{n_{2}, n_{3} \geq 0} \frac{q^{n_{2}+n_{3}+n_{2} n_{3}}}{(q)_{n_{3}}^{2}}=\frac{1}{(q)_{\infty}} \sum_{n \geq 0} \frac{q^{n}}{(q)_{n}^{2}\left(1-q^{n+1}\right)} \\
& =\frac{1}{(1-q)(q)_{\infty}} \sum_{n \geq 0} \frac{q^{n}}{\left(q^{2}, q\right)_{n}}=\frac{1}{(q)_{\infty}^{3}} \sum_{n \geq 0}(-1)^{n} q^{\frac{n(n+3)}{2}} .
\end{aligned}
$$

The identity for $\mathrm{F}(2,1,2)$ is slightly more involved in that it requires two applications of Heine's transformation. In particular, starting with (3.3),

$$
\begin{aligned}
F(2,1,2) & =\sum_{n_{1}, n_{3} \geq 0} \frac{q^{n_{1}+n_{3}}}{(q)_{n_{1}}^{2}(q)_{n_{3}}^{2}\left(q^{\left.1+n_{1}+n_{3}\right)_{\infty}}\right.}=\frac{1}{(q)_{\infty}} \sum_{n, m \geq 0} \frac{(q)_{n+m} q^{n+m}}{(q)_{n}^{2}(q)_{m}^{2}} \\
& =\frac{1}{(q)_{\infty}} \sum_{n \geq 0} \frac{q^{n}}{(q)_{n}} \sum_{m \geq 0} \frac{\left(0, q^{n+1}\right)_{m} q^{m}}{(q)_{m}^{2}} \stackrel{(3.6)}{=} \frac{1}{(q)_{\infty}^{2}} \sum_{n, m \geq 0} \frac{\left(q^{-n}\right)_{m} q^{n+m+m n}}{(q)_{n}^{2}} \\
& =\frac{1}{(q)_{\infty}^{2}} \sum_{n, m \geq 0} \frac{(-1)^{m} q^{n+\frac{m(m+1)}{2}}}{(q)_{n}(q)_{n-m}}=\frac{1}{(q)_{\infty}^{2}} \sum_{n, m \geq 0} \frac{(-1)^{m} q^{n+\frac{m(m+3)}{2}}}{(q)_{n}(q)_{n+m}} \\
& =\frac{1}{(q)_{\infty}^{2}} \sum_{m \geq 0} \frac{(-1)^{m} q^{\frac{m(m+3)}{2}}}{(q)_{m}} \sum_{n \geq 0} \frac{q^{n}}{\left(q^{m+1}, q\right)_{n}} \\
& \stackrel{13.6)}{=} \frac{1}{(q)_{\infty}^{4}} \sum_{n, m \geq 0}(-1)^{n+m} q^{\frac{n(n+1)}{2}+n m+\frac{m(m+3)}{2}}
\end{aligned}
$$

However,

$$
\begin{aligned}
& (1-q) \sum_{n, m \geq 0}(-1)^{n+m} q^{\frac{n(n+1)}{2}+n m+\frac{m(m+3)}{2}} \\
& =\sum_{\substack{n \geq 1 \\
m \geq 0}}(-1)^{n+m+1} q^{\frac{n(n-1)}{2}+n m+\frac{m(m+1)}{2}}-\sum_{\substack{n \geq 0 \\
m \geq 1}}(-1)^{n+m+1} q^{\frac{n(n-1)}{2}+n m+\frac{m(m+1)}{2}} \\
& =\sum_{n \geq 1}(-1)^{n+1} q^{\frac{n(n-1)}{2}}-\sum_{m \geq 1}(-1)^{m+1} q^{\frac{m(m+1)}{2}}=1+2 \sum_{n \geq 1}(-1)^{n} q^{\frac{n(n+1)}{2}}
\end{aligned}
$$

so that

$$
F(2,1,2)=\frac{1}{(1-q)(q)_{\infty}^{4}}\left(1+2 \sum_{n \geq 1}(-1)^{n} q^{\frac{n(n+1)}{2}}\right)
$$

Note that $F(1,1,3), F(1,1,4)$, and $F(2,1,3)$ are missing in the identities above.

Many of the identities for $F\left(r_{1}, r_{2}, r_{3}\right)$ follow from identities for $F\left(r_{1}, r_{2}\right)$. This is because (3.3) gives that $F\left(1, r_{2}, \ldots, r_{k}\right)=\frac{1}{(q)_{\infty}} F\left(r_{2}-1, r_{3}, \ldots, r_{k}\right)$, and trivially $F\left(r_{1}, r_{2}, \ldots, r_{k}\right)=$ $F\left(r_{k}, \ldots, r_{2}, r_{1}\right)$. Thus each identity for $F\left(1, r_{2}, \ldots, r_{k}\right)$ yields an identity for $F\left(1, r_{2}+\right.$ $\left.1, r_{3}, \ldots, r_{k}\right)$. In the following proposition, we record one particularly simple form of this iteration. 
Proposition 6.2. For $k \geq 2$,

$$
\sum_{n_{1}, n_{2}, \ldots, n_{k} \geq 0} \frac{q^{n_{1}+n_{2}+\cdots+n_{k}+n_{1} n_{2}+n_{2} n_{3}+\cdots+n_{k-1} n_{k}}}{(q)_{n_{1}}(q)_{n_{2}}^{2}(q)_{n_{3}}^{2} \cdots(q)_{n_{k-1}}^{2}(q)_{n_{k}}}=\frac{1}{(1-q)(q)_{\infty}^{k-1}}
$$

\section{Relations with Sum of Tails Identities}

Continuing from the previous section, we focus on the case $n_{1}=n_{2}=\cdots=n_{k}=1$, where $4 \leq k \leq 8$. Here we see certain Lambert series, quantum modular forms, and quasi-modular forms can appear. We have identities for $k=4,5$, and 6 , and conjectures for $k=7$ and 8 .

7.1. $k=4$. Using (3.3), it is easy to show that

$$
\sum_{n_{1}, n_{2}, n_{3}, n_{4} \geq 0} \frac{q^{n_{1}+n_{2}+n_{3}+n_{4}+n_{1} n_{2}+n_{2} n_{3}+n_{3} n_{4}}}{(q)_{n_{1}}(q)_{n_{2}}(q)_{n_{3}}(q)_{n_{4}}}=\frac{1}{(q)_{\infty}^{2}} \sum_{n_{2}, n_{3} \geq 0} q^{n_{2}+n_{3}+n_{2} n_{3}}=\frac{q^{-1}}{(q)_{\infty}^{2}} \sum_{n \geq 1} \frac{q^{n}}{1-q^{n}} .
$$

To see the connection with the identities for $k \geq 5$ and sums of tails identities, we note that we can also write

$$
\frac{1}{(q)_{\infty}} \sum_{n \geq 1} \frac{q^{n}}{1-q^{n}}=\sum_{n \geq 0}\left(\frac{1}{(q)_{\infty}}-\frac{1}{(q)_{n}}\right)=\frac{1}{(q)_{\infty}}-1+\sum_{n \geq 1}\left(\frac{1}{(q)_{\infty}}-\frac{1}{(q)_{n}}\right) .
$$

7.2. $k=5$. Again by (3.3), we have

$$
\sum_{n_{1}, n_{2}, n_{3}, n_{4}, n_{5} \geq 0} \frac{q^{n_{1}+n_{2}+n_{3}+n_{4}+n_{5}+n_{1} n_{2}+n_{2} n_{3}+n_{3} n_{4}+n_{4} n_{5}}}{(q)_{n_{1}}(q)_{n_{2}}(q)_{n_{3}}(q)_{n_{4}}(q)_{n_{5}}}=\frac{1}{(q)_{\infty}^{2}} \sum_{n \geq 0} \frac{q^{n}}{(q)_{n}\left(1-q^{n+1}\right)^{2}} .
$$

We note that the series has a straight-forward combinatorial interpretation. In particular,

$$
\sum_{n \geq 0} \frac{q^{n+1}}{(q)_{n}\left(1-q^{n+1}\right)^{2}}=\sum_{n \geq 0} t(n) q^{n}
$$

where $t(n)$ is the sum of the numbers of times that the largest part appears in each partition of $n$, which is reminiscent of Andrews celebrated smallest parts partition function [6]. The function $t(n)$ was studied for its asymptotic properties in [20]

It turns out this series has a much more interesting representation,

$$
\begin{aligned}
& \frac{1}{(q)_{\infty}^{2}} \sum_{n \geq 0} \frac{q^{n}}{(q)_{n}\left(1-q^{n+1}\right)^{2}}=\frac{q^{-1}}{(q)_{\infty}^{2}} \sum_{\substack{n \geq 1 \\
m \geq 0}} \frac{q^{n+n m}}{(q)_{n}}=\frac{q^{-1}}{(q)_{\infty}^{2}} \sum_{m \geq 0}\left(-1+\frac{1}{\left(q^{1+m}\right)_{\infty}}\right) \\
& =\frac{q^{-1}}{(q)_{\infty}^{3}} \sum_{m \geq 0}\left((q)_{m}-(q)_{\infty}\right)=-\frac{q^{-1}}{2(q)_{\infty}^{3}} \sum_{n \geq 1} n\left(\frac{12}{n}\right) q^{\frac{n^{2}-1}{24}}-\frac{q^{-1}}{(q)_{\infty}^{2}} \sum_{n \geq 1} \frac{q^{n}}{1-q^{n}}+\frac{q^{-1}}{2(q)_{\infty}^{2}}
\end{aligned}
$$

where the final equation follows by Theorem 2 of [33] and the discussion leading up to it. We note that upon ignoring the factor of $\frac{q^{-1}}{(q)_{\infty}^{3}}$, this final series was an essential component in Zagier's study [33] of a "strange identity" for Kontsevich's function and Zagier's construction of prototypical examples of quantum modular forms [34]. 
7.3. $k=6$. In this case, we find that

$$
\sum_{n_{1}, n_{2}, n_{3}, n_{4}, n_{5}, n_{6} \geq 0} \frac{q^{n_{1}+n_{2}+n_{3}+n_{4}+n_{5}+n_{6}+n_{1} n_{2}+n_{2} n_{3}+n_{3} n_{4}+n_{4} n_{5}+n_{5} n_{6}}}{(q)_{n_{1}}(q)_{n_{2}}(q)_{n_{3}}(q)_{n_{4}}(q)_{n_{5}}(q)_{n_{6}}}=\frac{1}{(q)_{\infty}^{2}} \sum_{n, m \geq 0} \frac{q^{n+m+n m}}{(q)_{n+1}(q)_{m+1}} .
$$

We mention in passing that the double sum appears to have a partition theoretic interpetation and matches entry A179862 of OEIS. More interesting is how this series reduces. Again by (3.3), we have

$$
\begin{aligned}
& \frac{1}{(q)_{\infty}^{2}} \sum_{n, m \geq 0} \frac{q^{n+m+n m}}{(q)_{n+1}(q)_{m+1}}=\frac{q^{-1}}{(q)_{\infty}^{2}} \sum_{n, m \geq 1} \frac{q^{n m}}{(q)_{n}(q)_{m}}=\frac{q^{-1}}{(q)_{\infty}^{2}} \sum_{n \geq 1} \frac{1}{(q)_{n}}\left(\frac{1}{\left(q^{n}\right)_{\infty}}-1\right) \\
& =\frac{q^{-1}}{(q)_{\infty}^{2}} \sum_{n \geq 1}\left(\frac{1}{(q)_{\infty}}-\frac{1}{(q)_{n}}+\frac{q^{n}}{\left(1-q^{n}\right)(q)_{\infty}}\right)=\frac{q^{-1}}{(q)_{\infty}^{3}} \sum_{n \geq 1} \frac{q^{n}}{1-q^{n}}+\frac{q^{-1}}{(q)_{\infty}^{2}} \sum_{n \geq 1}\left(\frac{1}{(q)_{\infty}}-\frac{1}{(q)_{n}}\right) \\
& =\frac{q^{-1}}{(q)_{\infty}^{3}} \sum_{n \geq 1} \frac{q^{n}}{1-q^{n}}-\frac{q^{-1}}{(q)_{\infty}^{2}}\left(\frac{1}{(q)_{\infty}}-1\right)+\frac{q^{-1}}{(q)_{\infty}^{2}} \sum_{n \geq 0}\left(\frac{1}{(q)_{\infty}}-\frac{1}{(q)_{n}}\right) \\
& =\frac{2 q^{-1}}{(q)_{\infty}^{3}} \sum_{n \geq 1} \frac{q^{n}}{1-q^{n}}-\frac{q^{-1}}{(q)_{\infty}^{3}}+\frac{q^{-1}}{(q)_{\infty}^{2}},
\end{aligned}
$$

where the final equality follows directly from Theorem 2 of [7] with $a=0$ and $b=c$.

7.4. $k=7$. Here we have a conjectural identity:

$$
\begin{gathered}
\sum_{n_{1}, n_{2}, n_{3}, n_{4}, n_{5}, n_{6}, n_{7} \geq 0} \frac{q^{n_{1}+n_{2}+n_{3}+n_{4}+n_{5}+n_{6}+n_{7}+n_{1} n_{2}+n_{2} n_{3}+n_{3} n_{4}+n_{4} n_{5}+n_{5} n_{6}+n_{6} n_{7}}}{(q)_{n_{1}}(q)_{n_{2}}(q)_{n_{3}}(q)_{n_{4}}(q)_{n_{5}}(q)_{n_{6}}(q)_{n_{7}}} \\
=\frac{q^{-1}}{(1-q)(q)_{\infty}^{4}}\left(\sum_{m \geq 1}(-3 m+1)(-1)^{m} q^{\frac{3 m^{2}+m}{2}}+\sum_{m \leq-1}(3 m+2)(-1)^{m} q^{\frac{3 m^{2}+m}{2}}\right) .
\end{gathered}
$$

The infinite series on the right-hand side is a quantum modular form. We also give another conjectural identity in the form of sum of tails

$$
\begin{aligned}
& \sum_{n_{1}, n_{2}, n_{3}, n_{4}, n_{5}, n_{6}, n_{7} \geq 0} \frac{q^{n_{1}+n_{2}+n_{3}+n_{4}+n_{5}+n_{6}+n_{7}+n_{1} n_{2}+n_{2} n_{3}+n_{3} n_{4}+n_{4} n_{5}+n_{5} n_{6}+n_{6} n_{7}}}{(q)_{n_{1}}(q)_{n_{2}}(q)_{n_{3}}(q)_{n_{4}}(q)_{n_{5}}(q)_{n_{6}}(q)_{n_{7}}} \\
& =\frac{q^{-1}}{(1-q)(q)_{\infty}^{4}}\left(-1+\sum_{n \geq 1} \frac{q^{n}}{1-q^{n}}(q)_{\infty}+\sum_{n \geq 0}\left((q)_{n}-(q)_{\infty}\right)+(q)_{\infty}\right) .
\end{aligned}
$$

7.5. $k=8$. Lastly we offer the following conjectural identity,

$$
\begin{gathered}
\sum_{n_{1}, n_{2}, n_{3}, n_{4}, n_{5}, n_{6}, n_{7}, n_{8} \geq 0} \frac{q^{n_{1}+n_{2}+n_{3}+n_{4}+n_{5}+n_{6}+n_{7}+n_{8}+n_{1} n_{2}+n_{2} n_{3}+n_{3} n_{4}+n_{4} n_{5}+n_{5} n_{6}+n_{6} n_{7}+n_{7} n_{8}}}{(q)_{n_{1}}(q)_{n_{2}}(q)_{n_{3}}(q)_{n_{4}}(q)_{n_{5}}(q)_{n_{6}}(q)_{n_{7}}(q)_{n_{8}}} \\
=\frac{q^{-2}}{(q)_{\infty}^{3}}\left(\left(\frac{1}{(q)_{\infty}}-1\right) \sum_{n \geq 1} \frac{n q^{n}}{1-q^{n}}\right) .
\end{gathered}
$$

We leave it as an open question to determine the behavior for general $k$. 


\section{Principal SUbSPaCES AND Infinite Jet SCHEMES}

In this part we require some familiarity with vertex algebras (especially lattice vertex algebras) and principal subspaces as developed in [17, 18, 13, 26].

We first form a lattice vertex algebra $V_{L}$ on the integral lattice $L=\mathbb{Z} \beta_{1}+\cdots+\mathbb{Z} \beta_{k}$, such that $\left(\beta_{i}, \beta_{i+1}\right)=1$, for $i=1, \ldots, k-1$, and zero otherwise. This is a non-degenerate even lattice for $k$ even. For $k$ odd it is degenerate with 1-dimensional radical subspace. For simplicity of exposition we shall ignore this degeneracy and consider only $k$ even here. We consider the principal subspace [26]

$$
W_{L}=\left\langle e^{\beta_{1}}, \ldots, e^{\beta_{k}}\right\rangle \subset V_{L}
$$

generated by $e^{\beta_{i}}$. Using tools of vertex algebras one can show that $W_{L}$ admits a nice monomial basis. For instance, for $k=2$, we get

$$
v=\beta_{2}\left(-j_{1}^{(2)}\right) \cdots \beta_{2}\left(-j_{n_{2}}^{(2)}\right) \beta_{1}\left(-j_{1}^{(1)}\right) \cdots \beta_{1}\left(-j_{n_{1}}^{(1)}\right),
$$

where $j_{k}^{(i)} \geq 1$ and $j_{i}^{(2)}>n_{1}$. Defining $\operatorname{deg}(v):=\sum_{i=1}^{n_{1}} j_{i}^{(1)}+\sum_{i=1}^{n_{2}} j_{i}^{(2)}$, the character of $W_{L}$ can be computed directly from this basis as

$$
\sum_{n_{1}, n_{2} \geq 0} \frac{q^{\left(n_{1}+1\right) n_{2}}}{(q)_{n_{2}}} \frac{q^{n_{1}}}{(q)_{n_{1}}}
$$

which is precisely $F(1,1)$. More generally, results from [26, 28] give:

Proposition 8.1. We have $F(\underbrace{1, \ldots, 1}_{\mathrm{k}-\text { times }})=\operatorname{ch}\left[W_{L}\right](q)$.

This formula can be interpreted in the language of infinite jet schemes. Consider an affine scheme $X$ and the $m$-th jet scheme $J_{m} X$ of $X$. This system has a projective limit in the category of schemes $J_{\infty} X:=\lim _{\leftarrow} J_{n} X$ called the arc space of the infinite jet scheme of $X$. Consider now (here $k \geq 2$ )

$$
R:=\mathbb{C}\left[x_{1}, \ldots, x_{k}\right] /\left(x_{1} x_{2}, \ldots, x_{k-1} x_{k}\right)
$$

and let $X=\operatorname{Spec} R$. Then the coordinate ring $J_{\infty} R=\mathbb{C}\left[J_{\infty} X\right]$ has a commutative vertex algebra structure (see [8, 27] for instance). For general vertex algebras we have a surjective morphism from $\mathbb{C}\left[J_{\infty} X\right]$ to $\operatorname{gr}(V)$ but for several examples of "nilpotent" vertex algebras, as well as some rational vertex algebras, this map is an isomorphism [23] (see also [27, 9, 16]). For instance, using the presentation of $W_{L}$ and the definition of $J_{\infty} R$ it is easy to see using presentation results from [26, 28, 22, 23] that

$$
W_{L} \cong J_{\infty} R
$$

as graded commutative vertex algebras. Therefore the Hilbert series of the arc space of $X$ satisfies

$$
H S_{q}\left(J_{\infty} X\right)=\operatorname{ch}\left[W_{L}\right](q) .
$$

Our results in Section 7 provide a completely new combinatorial aspect of these infinite jet schemes. 


\section{Final COMments}

In this section we present a few problems which need to be further addressed. First, there is a need for better understanding of the identities in Section 7. We still do not understand the nature of the $q$-series appearing on the "product" side. Although for $k=3$ and $k=8$ they are essentially modular, for $k=5$ and $k=7$ they behave as quantum modular forms, and for $k=2,4$, and 6 they are neither modular nor quantum (or false). Further connections with the sum of tails remains unclear to us.

We would also like to gain a better understanding of the combinatorics behind Conjecture 5.2 as there is already rich combinatorics governing monomial bases of basic $C_{n}^{(1)}$-modules [29].

9.1. Further $q$-series identities. In another direction, we can slightly modify the quadratic form in $F\left(r_{1}, \ldots, r_{k}\right)$ by adding the term $n_{k} n_{1}$ so that the summation is over the "circle". This way we can produce additional interesting identities. For instance, for $r_{1}=\cdots=r_{k}=1$ and $k=3$, we get an identity for a Ramanujan's fifth order mock theta function

$$
\sum_{n_{1}, n_{2}, n_{3} \geq 0} \frac{q^{n_{1}+n_{2}+n_{3}+n_{1} n_{2}+n_{2} n_{3}+n_{3} n_{1}}}{(q)_{n_{1}}(q)_{n_{2}}(q)_{n_{3}}}=\frac{1}{(q)_{\infty}} \sum_{n \geq 0} \frac{q^{n}}{\left(q^{n+1}\right)_{n+1}} .
$$

This follows directly from Euler's identity and $\sum_{m \geq 0}^{\infty} q^{m(n+1)} \frac{(q)_{n+m}}{(q)_{m}}=\frac{(q)_{n}}{\left(q^{n+1}\right)_{n+1}}$ [5. Theorem 3.3]. For $k=5$, we conjecture an elegant (quasi)-modular identity analogous to the $k=8$ case in Section 7,

$$
\sum_{n_{1}, n_{2}, n_{3}, n_{4}, n_{5} \geq 0} \frac{q^{n_{1}+n_{2}+n_{3}+n_{4}+n_{5}+n_{1} n_{2}+n_{2} n_{3}+n_{3} n_{4}+n_{4} n_{5}+n_{5} n_{1}}}{(q)_{n_{1}}(q)_{n_{2}}(q)_{n_{3}}(q)_{n_{4}}(q)_{n_{5}}}=\frac{q^{-1}}{(q)_{\infty}^{2}} \sum_{n \geq 1} \frac{n q^{n}}{1-q^{n}} .
$$

Since both expressions on the right-hand side are mock it would be interesting to see whether this persists in

9.2. Identities with higher order poles. For $r_{1}=\cdots=r_{k}=2$ and $k \geq 3$, we expect

$$
\frac{\sum_{n \geq 0}(-1)^{n k} q^{\frac{k}{2} n(n+1)}}{(q)_{\infty}^{k}}=\sum_{n_{1}, n_{2}, \ldots, n_{k} \geq 0} \frac{q^{\sum_{i=1}^{k-1} n_{i} n_{i+1}+n_{k} n_{1}+\sum_{i=1}^{k} n_{i}}}{(q)_{n_{1}}^{2}(q)_{n_{2}}^{2} \cdots(q)_{n_{k}}^{2}}
$$

again alternating between false identities for $k$ odd, and modular identities for $k$ even (observe, $\left.\sum_{n \geq 0} q^{\frac{k}{2} n(n+1)}=\frac{1}{2} \sum_{n \in \mathbb{Z}} q^{\frac{k}{2} n(n+1)}\right)$. Presumably, this can be proven by slight adjustments along the lines of [21, Theorem 5.5].

\section{REFERENCES}

1. A. K. Agarwal, G. E. Andrews, and D. E. Bressoud, The Bailey lattice, J. Indian Math. Soc. (N.S.) 1987, (1988) 57-73

2. G. E. Andrews, q-series: their development and application in analysis, number theory, combinatorics, physics, and computer algebra, Volume 66 of CBMS Regional Conference Series in Mathematics. Published for the Conference Board of the Mathematical Sciences, Washington, DC; by the American Mathematical Society, Providence, RI, 1986.

3. G. E. Andrews, Partitions and Durfee dissection, American Journal of Mathematics, 101(3), 735-742.

4. G. E. Andrews, Hecke modular forms and the Kac-Peterson identities, Trans. Amer. Math. Soc. 28336 (1984) 451-458.

5. G. Andrews, The theory of partitions, Cambridge University Press, Cambridge, 1998. 
6. G. E. Andrews. The number of smallest parts in the partitions of n. J. Reine Angew. Math., 624: (2008) pp. $133-142$.

7. G. E. Andrews, J. Jiménez-Urroz, and K. Ono. q-series identities and values of certain L-functions. Duke Math. J., 108(3): (2001) pp. 395-419.

8. T. Arakawa, Introduction to $W$-algebras and their representation theory, In Perspectives in Lie theory, pp. 179-250. Springer, Cham, 2017.

9. T. Arakawa and A. Linshaw, Singular support of a vertex algebra and the arc space of its associated scheme, arXiv: 1804.01287.

10. W. N. Bailey, Identities of the Rogers-Ramanujan type, Proc. London Math. Soc. 50 (1948) 1-10.

11. N. Bartlett and O. Warnaar, Hall? Littlewood polynomials and characters of affine Lie algebras, Advances in Mathematics 285 (2015): 1066-1105.

12. K. Bringmann, C. Calinescu, A. Folsom, and S. Kimport, Graded dimensions of principal subspaces and modular Andrews-Gordon-type series, Communications in Contemporary Mathematics, 16 (2014), 1350050 .

13. C. Calinescu, J. Lepowsky and A. Milas, Vertex-algebraic structure of the principal subspaces of level one modules for the untwisted affine Lie algebras of types A, D, E, Journal of Algebra 323 (2010): 167-192.

14. S. Cecotti, A. Neitzke, and C. Vafa. R-twisting and $4 d / 2 d$ correspondences, arXiv:1006.3435 (2010).

15. C. Cordova, S.-H. Shao, Schur Indices, BPS Particles, and Argyres-Douglas Theories, Journal of High Energy Physics 2016, no. 1 (2016): 40.

16. J. van Ekeren and R. Heluani, Chiral Homology of elliptic curves and Zhu's algebra, arXiv:1804.00017.

17. B. Feigin and A. Stoyanovsky, Quasi-particles models for the representations of Lie algebras and geometry of flag manifold, (1993) hep-th/9308079.

18. B. Feigin, and E. Feigin, Principal subspace for the bosonic vertex operator $\phi_{2 m}(z)$ and Jack polynomials, Advances in Mathematics 206 (2006): 307-328.

19. G. Gasper and M. Rahman, Basic hypergeometric series, Encyclopedia of Mathematics and its Applications volume 96, Cambridge University Press, second edition (2004).

20. P. J. Grabner and A. Knopfmacher, Analysis of some new partition statistics. Ramanujan J., 12(3): (2006) pp. 439-454.

21. C. Jennings-Shaffer and A.Milas, On $q$-series identities for false theta series, arXiv.2001.11368.

22. H. Li, Some remarks on associated varieties of vertex operator superalgebras, arXiv: 2007.04522, (2020).

23. H. Li, Equivariant oriented cohomology, jet schemes and vertex operator superalgebras, $\mathrm{PhD}$ thesis (UAlbany), in progress.

24. J. Lovejoy, Lacunary partition functions, Math. Res. Lett. 9 (2-3), 191-198, 2002.

25. A. Milas, On certain automorphic forms associated to rational vertex operator algebras, in "Moonshine: The First Quarter Century and Beyond", Edinburgh 2004, London Mathematical Society Lecture Notes Series 372 (2010): 330.

26. A. Milas and M. Penn, Lattice vertex algebras and combinatorial bases: general case and $W$-algebras, New York Journal of Mathematics 18 (2012): 621-650.

27. A. Moreau, Lecture notes on vertex algebras and associated varieties; available at http://math.univ-lille1.fr/ amoreau/LesDiablerets_Associated_Varieties-jan2019.pdf.

28. M. Penn, Lattice vertex superalgebras, I: Presentation of the principal subalgebra, Communications in Algebra 42, no. 3 (2014): 933-961.

29. M. Primc and T. Šikić, Combinatorial bases of basic modules for affine Lie algebras $C_{n}^{(1)}$, Journal of Mathematical Physics, 57(9), (2018) 091701.

30. L. J. Slater. A new proof of Rogers's transformations of infinite series, Proc. London Math. Soc. (2), no. 53 (1951), 460-475

31. A.V. Stoyanovsky, Lie algebra deformations and character formulas, Functional Analysis and Its Applications 32, no. 1 (1998): 66-68.

32. O. Warnaar and W. Zudilin, Dedekind's $\eta$-function and Rogers-Ramanujan identities, Bulletin of the London Mathematical Society 44, no. 1 (2012): 1-11.

33. D. Zagier. Vassiliev invariants and a strange identity related to the Dedekind eta-function. Topology, 40(5): (2001) pp. 945-960.

34. D. Zagier. Quantum modular forms. In Quanta of maths, vol. 11 of Clay Math. Proc., pp. 659-675. Amer. Math. Soc., Providence, RI (2010). 
Department of Mathematics, University of Denver, 2390 S. York St. Denver, CO 80208

Email address: christopher.jennings-shaffer@du.edu

Department of Mathematics and Statistics, SUNY-Albany, Albany, Ny 12222, USA

Email address: amilas@albany.edu 